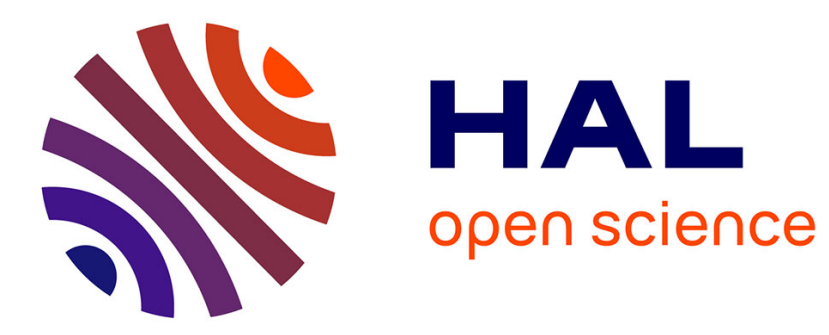

\title{
Prière et charité dans la Compagnie du Saint-Sacrement (1629-1667)
}

\author{
Alain Tallon
}

\section{To cite this version:}

Alain Tallon. Prière et charité dans la Compagnie du Saint-Sacrement (1629-1667). Histoire, économie et société, 1991, 10 (3), pp.331-343. 10.3406/hes.1991.1604 . hal-02056857

\section{HAL Id: hal-02056857 https://hal.sorbonne-universite.fr/hal-02056857}

Submitted on 4 Mar 2019

HAL is a multi-disciplinary open access archive for the deposit and dissemination of scientific research documents, whether they are published or not. The documents may come from teaching and research institutions in France or abroad, or from public or private research centers.
L'archive ouverte pluridisciplinaire $\mathbf{H A L}$, est destinée au dépôt et à la diffusion de documents scientifiques de niveau recherche, publiés ou non, émanant des établissements d'enseignement et de recherche français ou étrangers, des laboratoires publics ou privés. 


\title{
Prière et charité dans la Compagnie du Saint-Sacrement (1629-
} 1667)

Alain Tallon

\begin{abstract}
Abstract The Company of Holy Sacrement is very well-known, mostly owing to its numerous charity works in XVIIth century France. But its members also wanted to develop a special «spirit» and to encourage special devotions among themselves. That's why prayer and charity are the two points an which the devout's life is focused. Besides, the many common points between their praying and their charity works are noteworthy : the taste for secrecy, the appeal to individual conscience, and mostly the will to spiritualize prayer as well as charity, which finally meet, if not merge. But this balance soon breaks in favour of contemplation, which leaves action behind in the devout's ideal. The Company's failure at getting rid of the old dichotomy between action and contemplation brings to its final failure.
\end{abstract}

\section{Résumé}

Résumé La Compagnie du Saint-Sacrement est surtout célèbre pour toutes les œuvres charitables qu'elle a mises en place dans la France du XVIle siècle. Mais les confrères ont aussi voulu créer un "esprit» particulier et encourager en leur sein certaines pratiques de dévotion. Prière et charité sont donc les deux pôles de la vie du dévot. On peut noter d'ailleurs bien des points communs dans leur pratique de l'oraison et de l'action charitable : même goût du secret, même appel à la conscience de chacun, et surtout même volonté de spiritualiser la prière comme la charité, qui finissent par se rejoindre, sinon de fondre. Mais cet équilibre se rompt très vite, et la contemplation prend la première place devant l'action dans l'idéal dévot. L'échec de la Compagnie à sortir du vieux dilemne action/contemplation détermine son échec final.

\section{Citer ce document / Cite this document :}

Tallon Alain. Prière et charité dans la Compagnie du Saint-Sacrement (1629-1667). In: Histoire, économie et société, 1991, 10 année, $n^{\circ} 3$. Prières et charité sous l'Ancien Régime. pp. 331-343.

doi : $10.3406 /$ hes. 1991.1604

http://www.persee.fr/doc/hes_0752-5702_1991_num_10_3_1604

Document généré le 14/09/2015 


\title{
PRIERE ET CHARITE DANS LA COMPAGNIE DU SAINT-SACREMENT (1629-1667)
}

\author{
par Alain TALLON
}

\section{Résumé}

La Compagnie du Saint-Sacrement est surtout célèbre pour toutes les Ouvres charitables qu'elle a mises en place dans la France du XVIIe siècle. Mais les confrères ont aussi voulu créer un «esprit» particulier et encourager en leur sein certaines pratiques de dévotion. Prière et charité sont donc les deux pôles de la vie du dévot. On peut noter d'ailleurs bien des points communs dans leur pratique de l'oraison et de l'action charitable : même goût du secret, même appel à la conscience de chacun, et surtout même volonté de spiritualiser la prière comme la charité, qui finissent par se rejoindre, sinon de fondre. Mais cet équilibre se rompt très vite, et la contemplation prend la première place devant l'action dans l'idéal dévot. L'échec de la Compagnie à sortir du vieux dilemne action/contemplation détermine son échec final.

\section{Abstract}

The Company of Holy Sacrement is very well-known, mostly owing to its numerous charity works in XVIIth century France. But its members also wanted to develop a special «spirit» and to encourage special devotions among themselves. That's why prayer and charity are the two points an which the devout's life is focused. Besides, the many common points between their praying and their charity works are noteworthy : the taste for secrecy, the appeal to individual conscience, and mostly the will to spiritualize prayer as well as charity, which finally meet, if not merge. But this balance soon breaks in favour of contemplation, which leaves action behind in the devout's ideal. The Company's failure at getting rid of the old dichotomy between action and contemplation brings to its final failure.

Prière et charité au XVIIe siècle : il est difficile d'évoquer ce thème sans rencontrer la Compagnie du Saint-Sacrement. Créée en mars 1630, cette organisation secrète composée de laïcs et de prêtres séculiers connaît un succès prodigieux. En trente ans, elle essaime ses filiales dans une soixantaine de villes du royaume, et regroupe peutêtre quatre mille confrères. Son interdiction par le pouvoir le 13 décembre 1660 brise net une expansion qui allait devenir européenne. Si des compagnies de province subsistent jusqu'au XVIIIe siècle, la Compagnie, passée dans la clandestinité, disparaît comme organisation nationale autour de 1667 . Cette expérience est unique dans la France d'Ancien Régime : pendant trente-sept ans, une organisation a réussi à regrouper la majorité de l'élite dévote, en dehors de tout contrôle politique ou ecclésial. Dans un cadre chronologique qui va de la défaite du "parti dévot» aux débuts du règne personnel de Louis XIV, l'action charitable en France est presque monopolisée par les confrères : le seul exemple de saint Vincent de Paul, incarnation de la charité de ce 
siècle, est assez probant. Cet illustre membre de la Compagnie aurait-il pu réaliser toutes ses œuvres sans le concours actif des dévots ? Et Monsieur Vincent n'est qu'un exemple parmi tant d'autres personnalités du XVIIe siècle religieux.

Si la Compagnie du Saint-Sacrement est surtout connue comme l'organisatrice de plusieurs grandes œuvres charitables et religieuses, on s'est moins intéressé à la spiritualité propre aux confrères, et surtout au lien qui pouvait exister dans leur esprit entre prière et action charitable. Certes, cette étude est rendue difficile par la variété du milieu dévot : on y trouve des jansénistes, comme le duc de Liancourt, et des amis des Jésuites ; les docteurs de Sorbonne y rencontrent les familiers de Saint-Lazare. Par sa vocation même, qui est de regrouper les efforts de tous les catholiques militants de France, la Compagnie du Saint-Sacrement est difficile à étudier d'un point de vue spirituel. Son originalité s'impose cependant si on la compare avec d'autres milieux : celui des congrénanistes jésuites, par exemple, où la Compagnie recrute beaucoup, mais qui n'a pas exactement la même conception de la pratique dévote ${ }^{1}$. On a pu aussi montrer un lien entre la Compagnie du Saint-Sacrement et les compagnies de Pénitents. Mme Marguerite Pecquet écrit même : «Il me semble être absolument en droit d'avancer que la célèbre Compagnie du Saint-Sacrement n'est rien d'autre qu'une Compagnie de Pénitents, secrète ${ }^{2}$. Cette affirmation me semble excessive, car les pratiques expiatoires des Pénitents et leur ostentation, fondements même de la spiritualité pénitente, ne se retrouvent absolument pas à l'intérieur de la Compagnie du Saint-Sacrement. Cette dernière est tout à fait originale à plus d'un titre.

En effet, on ne peut réduire son rôle à celui d'une simple organisation charitable parmi d'autres, qui aurait laissé ses membres chercher ailleurs leur inspiration spirituelle. Au contraire, la Compagnie veut créer en son sein une véritable communauté d'esprit. Son point de départ est évidemment la pratique commune des œuvres charitables. Les confrères ont le sentiment de l'absolue nécessité des œuvres pour être sauvé : Renty, si souvent supérieur de la compagnie de Paris, cite Louis de Grenade dans une lettre du 30 août 1640 : «L'enfer est plain de bons desirs, et le Paradis de bonnes cuvres» ${ }^{3}$. Jean de Bernières, autre confrère, voit la marque de la grâce plus dans l'humble action du chrétien que dans «les visions, les revelations et les opérations de miracles. Travailler et souffrir pour Dieu vaut mieux que toutes les ecstases» ${ }^{4}$. Cette insistance sur le devoir chrétien d'agir dans le monde s'appuie sur une théologie des états, assez banale au XVIIe siècle : chacun doit se conformer à sa condition, et y faire son salut. Antoine Godeau, évêque de Grasse, déclare qu'un magistrat qui, oubliant ses devoirs, s'enfermerait chez lui pour se consacrer à la contemplation, pécherait aussi gravement qu'un ermite qui se mêlerait des affaires du siècles.

Cette théologie des états amène à se poser une première question : y a-t-il au sein de la Compagnie une séparation des tâches suivant la condition? Aux prêtres, la prière, et aux laïcs, laction charitable : cette répartition se retrouve dans beaucoup d'écrits de la Compagnie. Un confrère, Elie Laisné de la Marguerie, chargé de défendre les missions du Canada, écrit dans son livre que les prêtres sont riches en grâces célestes, mais pauvres en temporelles. Pour les laïcs, c'est le contraire. La prière des prêtres et la charité des laïcs permettent de rétablir «une très juste égalité, 
fondement asseuré de leur chrestienne société» ${ }^{6}$. La séparation des tâches semble simple, et mon étude pourrait s'arrêter là : les membres de la Compagnie respectent le caractère sacré du sacerdoce, seul habilité à traiter directement avec le divin, et cela en parfaite conformité avec l'esprit de la Réforme tridentine. Mais la réalité pratique au sein de la Compagnie prouve que cette belle théorie ne se vérifie pas toujours. Si les Memoires et instructions sur l'esprit de la Compagnie, envoyés en 1660 à toutes les compagnies de province, rappellent bien le thème classique de la séparation des tâches suivant les conditions, ils ajoutent : «Non pas que les mesmes applications ne conviennent pas à tous et que les religieux et ecclésiastiques ne travaillent avec bénédiction aux choses mixtes, ny que les laïcs ne s'appliquent à l'oraison, ne pratiquent la retraitte et la solitude et ne soient mesmes en éminent degré dans les exercices de mortification»?.

Notre étude a donc une raison d'être, puisque tous les membres de la Compagnie sont également appelés à la pratique de l'oraison et des bonnes œuvres. Mais comment vivent-ils cette double exigence ? Réunis avant tout pour agir, comment interprètent-ils leur action charitable sur le plan de la foi ?

\section{I - CHARITE ET SPIRITUALITE}

L'étude des rapports entre prière et charité au sein de la Compagnie du Saint-Sacrement passe nécessairement par une évaluation séparée de ces deux aspects de la vie du dévot. L'action charitable est bien connue, et a assuré sa réputation à la Compagnie. Mais cette réputation a souffert d'une certaine légende noire, en partie grâce au Tartuffe. Les dévots ne se contentent pas seulement d'aider les missionnaires ou de créer des hôpitaux. Ils jouent aussi un rôle de police spirituelle, qui exaspère déjà les contemporains. Mais pour un membre de la Compagnie du Saint-Sacrement, la persécution des protestants ou des libertins, la lutte pour la morale publique sont autant d'actions charitables envers le prochain, que l'on retire par force de son vice ou de son hérésie. Les Mémoires et instructions sur l'esprit de la Compagnie définissent ainsi le but de l'association : «entreprendre tout le bien possible et éloigner tout le mal possible

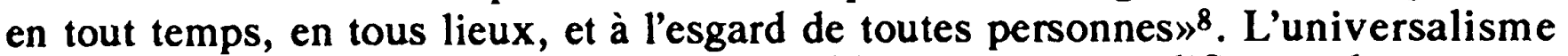
voulu par la Compagnie fait de son action un bloc, que nous qualifierons du nom que les dévots eux-même lui donnaient : celui de charité.

Pour évaluer cette action, il faut essayer d'en tracer un bilan. Il est impossible de le faire pour la compagnie de Paris, car les Annales de d'Argenson présentent de trop nombreux défauts. Elles sont tardives, puisque d'Argenson les rédige 34 ans après l'interdiction de la Compagnie. De plus, le compilateur a choisi ce qu'il fallait retenir ou laisser de côté en fonction de la situation politique et religieuse du moment. Les Annales peuvent donc parfois déformer notre vision de la Compagnie : par exemple, d'Argenson surestime beaucoup trop à mon avis l'action antiprotestante de la Compagnie, dans le but évident de plaire à Louis XIV.

Les registres de province sont plus fiables, même s'ils ne sont jamais complets. Pour Lyon et Marseille, on dispose d'extraits de registres, qui énumèrent les grandes 
réalisations et les projets 9 . A Lyon, le registre qui va de 1630 à 1668 , avec une interruption de 1633 à 1645 , comporte 65 mentions d'actions diverses. Le projet qui revient le plus souvent ( 15 fois) est celui de la Maison des Filles Pénitentes, seule grande réalisation de la compagnie de Lyon dans ces années-là. La seconde grande préoccupation est la décence morale et religieuse : interdiction de chansons «sales et indécentes», respect du Carême et des jours chômés, etc. Le secours matériel aux pauvres, aux malades ou aux prisonniers ne vient qu'après ces deux champs d'action. Les secours spirituels (c'est-à-dire par exemple l'instruction religieuse) arrivent en queue de la liste des réalisations lyonnaises, réalisations qui d'ailleurs restent bien modestes tant que dure la tutelle parisienne.

A Marseille, on retrouve le même schéma, quoiqu'avec quelques variantes : la lutte pour la décence publique et la morale religieuse est beaucoup plus accentuée qu'à Lyon. Les projets de fondations ne se limitent pas, comme dans la compagnie de Lyon, à une seule Maison de Filles Pénitentes, mais on trouve aussi la mention d'un Mont de Piété, d'un bureau de justice pour les pauvres, d'hôpitaux pour les incurables ou pour les forçats. Marseille organise aussi des missions, à la ville comme à la campagne. Tout ceci renforce l'idée d'une compagnie de Marseille beaucoup plus dynamique que son homologue lyonnais. Mais comme à Lyon, les mentions de secours temporels aux pauvres n'arrivent qu'après celles des projets de fondations et de combats pour l'ordre moral. Cependant, les secours temporels dépassent toujours les secours spirituels. Y a-t-il une contradiction avec les statuts de la Compagnie qui précisent que «les aumosnes purement temporelles ne regardans que la nourriture, entretiens et soulagement des pauvres [...] ne sont pas de l'esprit de la Compagnie»10 ? En réalité, les secours temporels fournis par les compagnies de Lyon ou de Marseille n'ont rien à voir avec les aumônes pratiquées dans les confréries classiques. Comme on le verra, ils sont d'une toute autre nature.

L'assistance individuelle, l'aumône quotidienne aux pauvres cèdent en effet le pas, au sein de la Compagnie, à des projets plus globaux. Non pas que les confrères oublient l'action au jour le jour. Au contraire, ils s'investissent physiquement dans les auvres charitables, et ne se contentent pas de vouloir régenter l'ordre public tout en demeurant dans l'ombre. Mais cette action se limite le plus souvent à la surveillance des œuvres déjà établies et au contrôle général des bonnes mœurs. Ainsi, chaque semaine, un laïc et un ecclésiastique sont délégués pour visiter les hôpitaux et les prisons de la ville, mais leur mission est autant de surveillance que d'assistance.

Les membres de la Compagnie inversent le schéma traditionnel de l'action dévote. Le bon confrère ne distribue plus une aumône quotidienne, mais va surtout visiter les pauvres, les hôpitaux, les prisons pour se rendre compte sur place des besoins. Ces besoins seront pris en charge par de grandes fondations, extérieures à la Compagnie. Les dévots insistent de plus en plus sur la nécessaire médiation d'une institution, et refusent de pratiquer l'aumône individuelle, qui conforte le mendiant dans son inaction, et amène le donateur à se glorifier de ses œuvres. En conséquence, les compagnies cessent assez vite de fournir de l'argent aux commis à la visite. A Lyon, «le 11 septembre 1647, fust résolut que celuy qui visiteroit les prisons y feroit l'aumosne à 
ses propres fraiz»11. A Marseille, on décide le 10 juillet 1643 «qu'on procureroit que les pauvres prisonniers heussent du pain chasque jour par la voye et du propre des personnes charitables qui voudroyent fournir à ceste despence» ${ }^{12}$. C'est seulement quand la misère devient trop épouvantable que les confrères acceptent de consacrer leur argent à des secours matériels : une lettre de 1663 d'un membre de la Compagnie à Monseigneur Pallu, l'informant de la situation, mentionne comme un cas exceptionnel le fait «qu'il a fallu appliquer tous les fonds de nostre Compagnie aux besoins profanes du prochain mourant a nos yeux» ${ }^{13}$. La Compagnie du Saint-Sacrement ne pratique qu'exceptionnellement les œuvres d'assistance classique. Elle ne veut pas être une simple organisation charitable de plus, mais tout faire partout «pour la gloire de Dieu et le bien du prochain», comme disent si souvent les dévots.

Dans la pratique, cela implique une insistance sur l'organisation de la charité publique. Rien de plus significatif sur la conception de la charité au sein de la Compagnie que ce texte, rédigé par Laisné de La Marguerie en faveur des missions du Canada : «il n'y a rien de si inutile, mal seant et nuisible à la police Chrestienne, qu'une Charité mal ordonnée ; c'est comme une marchandise meslée de contrebande pour le Ciel, c'est une perle parmi la boue, c'est une lumière en ténèbres; Dieu ne la cognoist pour sa fille aisnée, c'est une bastarde qui emprunte les habits et parures de sa legitime sœur, pour surprendre, si elle peut, la benediction qui ne luy est pas deue ; mais la pure et veritable charité, c'est comme une armée de bonnes cuvres bien rangée, chacune y garde son rang et ses files, sans que les unes s'empressent, n'y s'empeschent l'une l'autre, ni se foulent pour passer les unes devant les autres ${ }^{14}$. Cette conception conduit à la multiplication de sociétés publiques, dirigées par des confrères, qui s'efforcent de regrouper et d'ordonner les actions charitables soit dans un domaine particulier (le secours aux pauvres avec l'Hôpital Général, les missions étrangères avec la Société des Missions Etrangères de Paris), soit dans un secteur géographique précis, avec par exemple les compagnies de paroisse.

Mais l'organisation de la charité suppose aussi des priorités et des choix. Les dévots ne cessent de répéter qu'ils préferent la charité spirituelle à la charité temporelle. Un confrère, Louis Abelly, biographe de saint Vincent de Paul, définit ainsi la charité dans un manuel destiné aux pieux laïcs : elle est un mouvement raisonnable qui pousse à aimer le prochain et à lui procurer les vrais biens, c'est-à-dire son salut ${ }^{15}$. Mouvement raisonnable, car comme l'explique Laisné de La Marguerie, le chrétien qui fait l'aumône par pure compassion, sans réfléchir, ne fait pas une bonne œuvre. Cette pitié est en effet le propre des Infidèles, qui ne connaissent que les vertus naturelles et non les vertus chrétiennes ${ }^{16}$. Un vrai chrétien, avant de faire l'aumône, se demande ce qui sera le plus utile à son prochain, et la réponse est évidente : l'aider à se procurer son salut. Abelly insiste sur ce point : «Si vous voulez rendre vostre aumosne bien agréable à Dieu, tâchez de la faire en sorte qu'elle soit utile non seulement au soulagement du corps, mais aussi au salut de l'âme : ce point est d'une très grande importance et mérite que vous y apportiez bien de la considération» ${ }^{17}$. Et l'auteur donne quelques exemples : profiter d'une aumône pour catéchiser un pauvre, ou l'obliger à aller se confesser, offrir des livres de piété et des catéchismes, léguer de l'argent pour fonder 
des écoles ou des séminaires. On reconnaît là des activités typiques de la Compagnie : par exemple en mai 1652, la compagnie de Paris assemble dans un cimetière des refugiés des campagnes lorraines et picardes dévastées par la guerre, et leur fait donner en même temps des secours temporels et une instruction religieuse ${ }^{18}$.

L'œuvre charitable doit être occasion de salut. La compagnie de Lyon donne en 1667 une applicaton pratique à ce principe. On sait qu'à chaque séance, les confrères écrivaient sur des billets anonymes leurs propositions d'aumônes. La compagnie précise qu'«affin que les billets qui tendent au soulagement du prochain soient jugez de l'esprit de la Compagnie, il faut qu'ils ayent l'une des fins suivantes : 1) qu'il s'agisse de relever quelque pauvre particulier ou quelque pauvre famille tombez ou prestz à tomber dans une extrême nécessité, et que le bien qu'on leur peut faire les mette en estat de gaigner leur vie sans autre secours ; 2) pour retirer quelque fille ou femme de danger évident de se perdre ou la relever entièrement du vice si elle y est tombé ; 3) pour empescher la chute des chancelans en la foy sollicitez par les religionnaires ou malignes tentations ; 4) pour soulager tous ceux qui souffrent persécution pour la foy, et la 5) pour renvoyer chez eux les misérables et dépourvus d'appuys, pourveu qu'on scache qu'ils trouveront du bien ou du secours temporel et rettraite en leur pays». Les billets qui ne répondent pas à ces critères seront brûlés ${ }^{19}$.

Le salut qu'on doit procurer aux autres par l'aumône est conçu par les dévots comme une conversion brutale, un changement définitif d'état. Cela explique l'un des paradoxes de la Compagnie : elle qui est si tenace pour la plupart de ses cuvres, qui est capable de poursuivre dix ou vingt ans un projet, n'assure aucun «suivi» des pauvres auxquels elle a fait l'aumône. L'aide est ponctuelle, et si la conversion ne vient pas, c'est que le bénéficiaire est foncièrement mauvais. Il faut alors se détourner de lui. Un confrère exprime très bien cette idée : «Entretenez les victimes de Dieu, et ne servez point à engraisser les victimes du Diable. Aydez à la vie de tous ceux qui ne veulent de vie que pour servir à celuy qui la leur a donnée ; mais refusez-la hardiment à tous ces autres, en qui, apres les avoir examinez, ou fait examiner, vous ne voyez encore nulle disposition à en estre meilleurs» ${ }^{20}$. Ce choix de la charité spirituelle oblige les dévots à discerner parmi la masse des pauvres les damnés et les prédestinés, les bons et les mauvais.

Il amène aussi une dévalorisation certaine de l'aumône traditionnelle. Laisné de La Marguerie, chargé des missions étrangères au sein de la Compagnie, voit dans la prédominance des cuvres corporelles sur les spirituelles un des plus grands abus du siècle ${ }^{21}$. Il s'en plaint encore dans un autre ouvrage consacré aux missions du Levant : «Mais pourquoy mon Dieu, cette lumière de Charité Apostolique est-elle maintenant en la plupart si refroidie ? Je n'entends pas de cette charité qui s'appelle aumosne, qui n'en est qu'une des filles qui se fait adorer du monde plus que sa mère, qui est quelquefois aussi commune a tel Publicain ou adultère et usurier, qu'aux personnes vertueuses. Mais je parle de cette charité intérieure et divine qui juge des choses et agit par les principes de la haute sagesse, abnégative de sa vie, ses plaisirs et interests pour le service de Dieu, en la conversion des ames, non seulement de celles qui sont à nostre commodité près de nous [...] mais particulièrement des ames dispersées par le monde 
en peril d'une reprobation eternelle» ${ }^{22}$. La nécessité de spiritualiser leurs actions, pour utiliser une autre expression de Laisné de la Marguerie, conduit les dévots à les assimiler à l'autre œuvre spirituelle par excellence, la prière.

\section{II - LA PRIERE EST LA VRAIE CHARITE}

La Compagnie, si originale dans sa conception de la charité, semble l'être beaucoup moins pour la prière. Peu de choses dans ce domaine la distinguent des simples confréries. On retrouve la même idée d'une solidarité de tous les membres dans la prière, solidarité qui crée une communion des saints sur la terre, par une commune participation aux mérites de chacun. Dès sa création, la Compagnie insiste sur cette communauté, qui permet de déléguer à quelques uns la tâche de prier pour les autres : trois personnes par semaine sont ainsi choisies parmi les confrères «lune pour faire oraison sur quelqu'un des mystères de la vie de Notre-Seigneur, une autre toujours pour visiter le Saint-Sacrement de la part de la compagnie, et l'autre (pour prier) pour quelque nécessité publique, suivant l'ordre du Directeurs ${ }^{23}$. Ces prières faites au nom de toute la Compagnie sont bien peu originales.

L'ordre des prières dans les réunions est tout aussi banal. Toutes les assemblées s'ouvrent et se ferment par ces paroles : «Loué soit le Très Saint-Sacrement de l'Autel», devise de la Compagnie. Les autres prières utilisées pour le début de l'assemblée sont le Veni Creator et le Pange lingua A la fin de l'assemblée, on chante de même le psaume Laudate Dominum omnes gentes et le Gloria Patri. On y ajoute une antienne à la Vierge, une prière pour l'Eglise (Ecclesiae tuae quaesumus), pour le roi (Domine, salvum fac regem), pour la paix (Da pacem, Domine et Deus a quo sancta desideria ). Si un confrère est mort, on récite le $D e$ profundis et l'oraison $A b$ solve, Domine en son honneur. Si un autre est malade, il faut réciter l'Ave maris Stella et l'oraison pour les infirmes Deus salus aeterna credentium. On reprendra le Veni Creator et l'Ave maris Stella «pour quelque nécessité ou dévotion de la Compagnie». Enfin, après l'élection des officiers, les confrères entonnent le Te Deum ${ }^{24}$.

Cette énumération un peu fastidieuse permet de soulever un problème pratique : si les confrères respectent ainsi le rituel prévu par les statuts, les deux heures de réunion devaient être largement entamées par ces prières collectives. En fait, il y a eu une certaine inflation avec le temps : les statuts de Marseille, datés de 1639, contiennent nettement moins de prières que ceux de Poitiers, plus tardifs dans leur rédaction ${ }^{25}$. Cela s'explique par les ordres de la compagnie de Paris, qui demande souvent d'ajouter certaines prières, de façon exceptionnelle ou régulière. Le 16 juillet 1643, Marseille ajoute une oraison pour la paix aux prières de la compagnie, après avoir eu communication d'une résolution de Paris dans ce sens. Le 8 novembre 1645, Paris demande d'ajouter encore une prière pour la destruction des adversaires de l'Eglise et de leurs erreurs ${ }^{26}$. Néanmoins, les compagnies gardent leur autonomie, et peuvent ne pas lire certaines prières : c'est le cas à Lyon, où l'on note parmi les prières usuelles «lesquelles ne seront point leues pour n'estre pas observéez à Lyon» ${ }^{27}$. 
Pourquoi une Compagnie chargée avant tout de l'action charitable a-t-elle autant insisté sur ces exercices de piété ? La Compagnie cherche à créer une communauté d'esprit entre tous ses membres. Or, l'action charitable favorise plutôt la dispersion. Les tâches sont si écrasantes qu'elles sont souvent confiées à un petit comité restreint : quelques uns s'occupent de la lutte contre les duels, d'autres des missions étrangères, d'autres des projets d'hôpitaux, etc. La Compagnie du Saint-Sacrement risque de ne plus être qu'une fédération assez lâche de divers comités charitables spécialisés. C'est sans doute pour conjurer ce danger qu'un rituel assez lourd est créé pour les réunions. Mais l'aspect stéréotypé de ce rituel, l'absence de toute originalité posent un problème : la Compagnie, qui veut tout spiritualiser, n'a pas vraiment réussi à le faire pour le rituel de ses réunions. Cette absence de spontanéité et d'intériorité dans la prière commune des confrères oblige à étudier plus avant la conception même de la prière au sein de la Compagnie du Saint-Sacrement.

On retrouve dans ce domaine le même binôme d'opposition que pour la conception de la charité : les confrères établissent une hiérarchie de valeur entre l'oraison mentale et la prière vocale. Les témoignages de la faveur des confrères pour la première sont multiples. Je n'en citerai qu'un : François Renar, directeur de la compagnie de Paris mort en fonction, est présenté en exemple aux dévots dans une biographie officielle écrite par Abelly, autre confrère. Or, il pratique deux fois par jour l'oraison mentale, comme le décrit son biographe : «sa maniere d'Oraison estoit fort simple et affective ; il la commençoit toujours par une profonde demission de luy mesme devant Dieu, se prosternant d'esprit et de corps en sa presence» 28 . Cette idée de démission de soi conduit de la simple oraison mentale à sa version plus mystique, l'oraison passive.

Son théoricien le plus connu est Jean de Bernières, qui est en même temps le chef de la compagnie de Caen. Mais il n'est pas le seul au sein de la Compagnie à la pratiquer avec ferveur. Renty, le grand modèle des membres de la Compagnie, est lui aussi un adepte de cette pratique spirituelle. La biographie écrite par son directeur spirituel, le jésuite Saint-Jure, gomme un peu cet aspect là. Mais une religieuse amie de Renty écrivait après sa mort «qu'il ne pouvait plus dire, depuis 5 ou 6 ans, aucune prière vocale, sinon une petite Couronne de trois Pater et de douze Ave. Encore était-ce avec assez de peine, étant heureusement contraint de se laisser aller aux attraits puissants de l'amour divin, et d'écouter avec silence ce que l'Esprit-Saint lui disait au cœur» ${ }^{29}$. La prière idéale devient celle qui se passe absolument de mots, et se contente d'écouter. On comprend dans ces conditions que les confrères se soient contentés dans leurs réunions de formules toutes faites. Entre celles-ci et l'oraison mentale, voire passive, il n'y a rien ; aucune place n'est laissée pour une prière vocale spontanée.

Prière et charité sont toutes deux fortement spiritualisées au sein de la Compagnie du Saint-Sacrement. L'action doit se débarasser de tout caractère «temporel», comme disent les dévots, et surtout rester bien cachée. Ce thème du secret se retrouve quand les confrères doivent prier : il faut éviter la parole et se contenter de rentrer en soi, dans le secret de son cœur. Spirituel égale secret dans l'esprit des membres de la Compagnie. Pour la conception de la charité comme pour celle de la prière, les conséquences de cette équivalence spirituel-secret sont très importantes. 
Action et prière sont donc conçues sur le même modèle au sein de la Compagnie. Mais ce n'est pas le seul rapport. Une cuvre n'est vraiment bonne que si elle a été inspirée par Dieu. La prière doit précéder l'action. D'après un confrère toulousain, «elle doist estre un pourparler avec son Prince pour y prendre les ordres pour l'exécution des commissions qu'il nous donne» ${ }^{30}$. Cette image presque militaire du dévot qui va aux ordres dans la prière se retrouve chez d'autres membres de la Compagnie. Bernières établit une sorte de cercle dévot : «Les bonnes actions faites dans l'ordre de la grace entretiennent la contemplation, la devotion et l'interieur ${ }^{31}$. Mais pour les réaliser, il faut d'abord beaucoup prier. La prière nourrit l'action qui nourrit la prière.

Les devots ne parlent pas seulement de l'interaction entre prière et charité. Pour eux, ces deux réalités de la vie chrétienne peuvent se confondre. On trouve souvent sous la plume des confrères l'idée qu'exprime Renty que «toute auvre est oraison quand elle est faitte pour Dieu»32. Tout le monde connaît le mot de saint Vincent de Paul dans sa conférence du 31 juillet 1634 aux Filles de la Charité : «Mes filles, sachez que, quand vous quitterez l'oraison et la sainte messe pour le service des pauvres, vous n'y perdrez rien, puisque c'est aller à Dieu que servir les pauvres ; et vous devez regarder Dieu en leur personne». Mais quel est ce service des pauvres qui remplace la prière ? Le saint ajoute juste après : «Soyez doncques bien soigneuses de tout ce qui leur est nécessaire, et veillez particulièrement à l'aide que vous leur pouvez donner pour leur salut : qu'ils ne meurent pas sans les sacrements. Vous n'êtes pas là seulement pour leur corps, mais pour les aider à se sauver» ${ }^{33}$. L'acte charitable mis sur le même plan que la prière est donc avant tout spirituel. Leur finalité commune, le salut des âmes, permet cette identification.

Mais si la charité spirituelle devient prière, l'inverse est également vrai : la prière est en effet le moyen le plus noble peut-être de procurer le salut d'autrui. Laisné de la Marguerie dit même que c'est le seul qui corresponde à l'idéal d'universalisme que la Compagnie s'est imposé en matière de charité : «Prier Dieu est une œuvre spirituelle, et la seule de toutes par laquelle nous pouvons aumosner et faire bien à tout le monde» ${ }^{34}$. La spiritualisation de l'action arrive à son terme : la vraie charité chrétienne est le moins visible, le moins matériel de tous les actes, une prière.

La charité est prière, la prière est charité. Tout semble aller pour le mieux dans le meilleur des mondes dévots. Le moine contemplatif comme le laïc dévoué ont exactement le même rôle au regard de Dieu. Jean de Bernières résume la position de la Compagnie quand il écrit : «Agir, ou souffrir, ou prier m'est tout un quand l'ordre de Dieu y est $»^{35}$. Mais le prix à payer pour cet équilibre théorique risque de mettre ce dernier en danger. L'équilibre n'est en effet atteint que par la spiritualisation la plus poussée de la charité et de la prière. Or, cette opération implique aussi toute une thématique de l'anéantissement de soi, de passivité devant Dieu. On voit le danger qu'il y a de vouloir y inclure l'action charitable, comme le font les dévots. De plus, comment répartir son temps entre prière et charité, action et contemplation? La belle ordonnance qui se dégage des écrits des membres de la Compagnie s'effrite bien vite devant les contraintes quotidiennes de la vie dévote. 
Renty fournit un bon exemple d'un dévot contraint de choisir entre l'action charitable et la prière. Denis Amelote raconte dans la Vie d'une carmélite liée à Renty, la sœur Marguerite du Saint-Sacrement qu'il «a esté quelquefois en doute s'il ne falloit pas mieux qu'il s'appliquast à catechiser les pauvres, à quoy il travailloit avec un zele et une charité admirable, que de se retirer pour vaquer à la prière. Elle luy declara qu'il devoit faire tous les jours deux heures d'Oraison, et la preferer de telle sorte à ses Catechismes» ${ }^{36}$. Renty lui-même raconte à son directeur Saint-Jure un autre épisode où prière et charité entre en conflit : priant devant le Saint-Sacrement, un pauvre vient lui demander l'aumône. Situation scandaleuse pour un membre de la Compagnie, qui ne supporte pas que les églises soient le repaire des vagabonds. La compagnie de Grenoble, par exemple, décide en février 1652 de payer un suisse pour empêcher les pauvres de venir demander l'aumône à la cathédrale pendant l'office ${ }^{37}$. En bon dévot, Renty manifeste donc d'abord quelque mécontentement, puis il se reprend : «comme il nous faut bien souffrir avec patience les distractions intérieures, nous devons endurer de mesme les exterieures» ${ }^{38}$.

La charité devient distraction, conséquence logique de la spiritualisation exigée en tout par l'idéal dévot. La prière est à nouveau placée au-dessus de la charité. Il n'est plus question d'égalité entre ces deux pôles de la vie chrétienne. Car la spiritualisation de la prière passe aussi par l'abandon et la dévalorisation de tout exercice extérieur de piété. Certes, les dévots les pratiquent, mais il gardent l'idée que la seule vraie prière est celle qui se remarque le moins à l'extérieur, et surtout introduit directement à la contemplation de Dieu. Même la plus spirituelle des charités ne permet pas un tel anéantissement. Elle est donc comprise dans ce désinvestissement spirituel général, que les dévots font subir aux exercices extérieurs de piété comme aux œuvres de charité. «Fuyons la dévotion du temps, s'exclame un confrère, on se confesse, on se communie, on fait oraison, on fait quantité de bons exercices, tout cela est beau et bon : mais il n'y a point de solidité que dans la destruction de nous-mesmes ${ }^{39}$. Le beau modèle théorique construit par les dévots n'a pas résisté à l'épreuve pratique. Non pas que les confrères cessent leurs activités charitables et leurs exercices de piété, bien au contraire. Mais ils ont tendance à les dévaloriser. Il ne faut pas s'étonner de trouver sous la plume de ceux-là mêmes qui assimilaient l'action charitable à la prière, des dénonciations amères de la vanité des cuvres.

Bernières est sans doute celui qui établit le plus clairement une hiérarchie, prenant pour comparaison le monde de la Cour : «Dans la maison d'un Roy, les cuisiniers, boulengers et autres menus Officiers, travaillent et agissent plus qu'un Gentilhomme ordinaire de la Chambre, qui n'a qu'à estre dans la Chambre du Roy pour l'accompagner. Un favory fait encore moins, car il se repose dans le cabinet et ne fait que s'entretenir avec luy et recevoir ses caresses et luy en rendre de réciproques. Dans la maison de Dieu, ceux qui font la plus grande action ne sont pas les plus favorisez; ceux que Dieu caresse dans la contemplation, travaillent moins et toutefois plaisent le plus. Ne nous attachons point trop aux actions extérieures de Charité» ${ }^{40}$. Est-ce seulement un pur retour à l'opposition classique entre la noblesse de la contemplation et la bassesse de l'œuvre? Les confrères semblent avoir échoué dans leur volonté de sur- 
monter ce vieil antagonisme. Pourtant, leur effort n'a pas été sans résultat. Sans dépasser l'opposition, ils en ont déplacé le sens.

Grands lecteurs de l'Evangile, les confrères ne pouvaient manquer de réfléchir sur celui de Marthe et Marie, où le Christ, d'après l'interprétation traditionnelle, préfere la contemplation à l'action. Nous avons une longue lettre de Renty à Saint-Jure, datée d'août 1646, où il explique que cet évangile l'a longtemps tourmenté, spécialement le reproche du Christ à Marthe : "Turbaris erga plurima» (Luc, 10, 41). Il en concluait qu'il fallait se «séparer des choses extérieures et mesmes des intérieures, quoy que bonnes, qui n'estoient pas absolument necessaires, comme d'aller visiter et instruire les pauvres, lire ou écrire quelque chose de devotion, et autres semblables». C'est alors la fin de toute vie dévote active, et Renty n'a plus qu'à se réfugier dans la contemplation. Mais il a une illumination, pendant laquelle il lui est dit : «Non turbaris erga plurima ». Il découvre alors que «l'ouvrage extérieur se peut faire du bout des doigts, pendant que le cour jouit d'une alliance réelle des enfans avec leur Pere par l'esprit du Fils» ${ }^{41}$. Peu importe au fond l'action extérieure, pourvu que le dévot n'y attache aucune importance, l'effectue «du bout des doigts», et reste dans un état intérieur de permanente contemplation. La scission entre extérieur et intérieur, thème classique de la spiritualité du XVIIe siècle, est ici totale. L'unité entre prière et charité, si fortement marquée dans les écrits des confrères, est en fait un trompe-l'œil. La véritable prière n'a rien à voir avec ces exercices extérieurs, qu'il s'agisse d'actes de dévotion ou d'actions charitables.

Bernières a lui aussi fait un long commentaire de l'Evangile de Marthe et Marie. Il permet peut-être de foumir la clé de l'attitude des dévots : «O que les creatures me semblent estre une dure captivité à l'ame, et que Marie Madeleine me plaist dans son oisiveté ! Elle laisse tout pour ne se soucier que de son unique amour, et son oubli va si avant, qu'elle oublie mesme les cuvres de misericorde et qu'elle ne fait rien pour donner à manger au corps de Jesus ; elle se repaist elle-mesme de la veue des perfectons divines, et l'amour qu'elle a pour Jesus luy fait oublier Jesus. Car ses divines perfections l'occupent tellement quelle ne pense point à luy preparer à manger comme sa sœur. Mon ame, quand l'attrait à l'oraison vous tiendra liée, ne craignez point de negliger les choses temporelles ; vostre principale affaire, c'est d'estre dans l'actualité du pur amour» ${ }^{42}$. «L'amour qu'elle a pour Jésus luy fait oublier Jésus» : cette phrase explique bien toutes les contradictions de l'attitude des dévots. Ces contradictions procèdent de leur vision même du Christ.

On sait que le XVIIe siècle français est celui du christocentrisme. Les membres de la Compagnie ne font pas exception à la règle. Mais quel est ce Christ qu'ils choisissent de contempler et de prendre pour modèle ? C'est essentiellement le Christ de la Passion, homme de douleur qui se laisse crucifier ; l'Enfant-Jésus, dont la dévotion se propage au sein de la Compagnie sous l'influence de Renty et du Carmel de Beaune ; enfin Jésus présent au Saint-Sacrement. Dans les trois cas, c'est un Christ inactif que vénèrent les dévots. C'est aussi un Christ presque inhumain. Leur théologie de l'Incarnation est en effet presque nestorienne : d'un côté, le Christ de gloire, triomphant à la droite du Père ; de l'autre, un homme humilié, souffrant, ou chétif. 
L'Incarnation du Verbe se comprend presque exclusivement comme son humiliation. Il ne reste plus qu'à le contempler dans ces deux états extrêmes.

On comprend alors pourquoi la contemplation inactive est l'idéal des dévots. Elle est la seule véritable imitation de Jésus-Christ. L'activité est un mal nécessaire, un devoir imposé pour son salut. Mais sa valeur spirituelle est presque nulle. Faute d'une théologie de l'Incarnation adaptée, les membres de la Compagnie du Saint-Sacrement accomplissent les œuvres de Marthe en conservant l'esprit de Marie. Prière et charité doivent céder le pas à la seule œuvre véritable du chrétien, la contemplation.

\section{NOTES}

1 M. Louis Châtelier indique ainsi que les congrégations jésuites ont tendance à faire passer «les dévotions publiques avant l'homme intérieur", ce qui est l'exact contraire pour la Compagnie : Châtellier (L.), L'Europe des dévots, Paris, 1987, p. 163.

2 Pecquet (M.), «des Compagnies de Pénitents à la Compagnie du Saint-Sacrement» dans Dix-septième siècle, t. 69 (1965), p. 20.

3 Renty (G. de), Correspondance, éditée par R. Triboulet, Paris, 1978, p. 82.

4 Bernières-Louvigny (J. de), L'Intérieur chrestien ou la conformité intérieure que doivent avoir les Chrestiens avec Jésus-Christ 2e édition, Paris, Veuve Edme Martin, 1674, p.12.

5 Godeau (A.), L'idée d'un bon magistrat en la vie et en la mort de Monsieur de Cordes, conseiller au Chastelet de Paris, Paris, Antoine Vitré, 1645, pp. 57-58.

${ }^{6}$ Laisne de la Marguerie (E.), Les véritables motifs de Messieurs et Dames de la société de Nostre-Dame de Monreal pour la conversion des sauvages de la nouvelle France, s.1., 1643, p.9.

7 Guigue (G.) éd., Les papiers des dévots de Lyon : recueil de textes sur la Compagnie secrète du Saint-Sacrement, ses statuts, ses annales, la liste de ses membres 1630-1731, Lyon, 1922, p. 29.

8 Guigue (G.), éd., ibidem, p. 34.

9 On trouvera ces registres publiés dans Allier (R.), éd., Une société secrète au XVIIe siècle : la Compagnie du Très-Saint-Sacrement de l'autel à Marseille, Paris, 1909, 492 p. ; et dans Guigue (G.) éd., Les papiers des dévots de Lyon, op.cit., 245 p.

10 Guigue (G.), éd., Les papiers des dévots de Lyon, op.cit, p. 41.

11 Guigue (G.), ed., ibidem, p. 69.

12 Allier (R.), éd., La Compagnie du très-Saint-Sactement de l'autel à Marseille, op.cit., p. 55.

13 Archives des Missions étrangères de Paris, volume CXV, $\mathrm{f}^{\circ} 333$.

14 Laisné de la Marguerie (E.), Les véritables motifs, op.cit, pp. 53-54.

15 Abelly (L.), Addresse pour utilement procurer le salut des ames. Où sont proposées diverses pratiques propres à toutes sortes de personnes, pour exercer les auvres de charité envers le Prochain, Paris, George Josse, 1644, pp. 9-17.

16 Laisné de La Marguerie (E.), Les veritables motifs, op.cit, p. 39.

17 Abelly (L.), Addresse..., op.cit., p. 276. 
18 Argenson (R. de Voyer d'), Annales de la Compagnie du Saint-Sacrement éditées par dom Beauchet-Filleau, Paris-Poitiers, 1900, p. 127.

19 Guigue (G.), éd., Les papiers des dévots de Lyon, op.cit, p. 75.

20 Noulleau (J.B.), L'esprit du christianisme, tiré de cent paroles choisies de Jésus-Christ..., Paris, Charles du Mesnil, 1664, tome 1, p. 597.

21 Laisné de La Marguerie (E.), Les véritables motifs, op.cit, p. 49.

22 Laisné de La Marguerie (E.), Memoires et instructions chrestiennes sur le sujet des Missions estrangères, et particulierement de celles qui se font en Turquie et autres pays du Levant, Paris, Pierre de Bresche, 1644, pp. 39-40.

23 Argenson (R. de Voyer d'), Annales..., op.cit, p. 15.

24 Tout ceci se trouve dans les statuts de Poitiers : Argenson (R. de Voyer d'), Annales..., op.cit., pp. 296297.

25 Allier (R.) éd., La Compagnie du Très-Saint-Sacrement de l'autel à Marseille..., op.cit, p.21.

26 Rebelliau (A.) éd., La Compagnie secrète du Saint-Sacrement : lettres du groupe parisien au groupe marseillais 1639-1662, Paris, 1908, pp. 35 et 59.

27 Guigue (G.) éd., Les papiers des dévots de Lyon..., op.cit, p.9.

28 Abelly (L.), L'idée d'un veritable prestre de l'Eglise de Jésus-Christ et d'un fidele directeur des ames, exprimée en la vie de Mr Renar..., Paris, Florentin Lambert, s.d. (1659?), pp. 16-17.

29 Elisabeth de Baillou de l'Enfant-Jésus, «Recueil de quelques grâces et vertus singulières que j'ai remarquées en Monsieur de Renty" édité par A. Bessières dans La vie spirituelle, t. 33 (1932), p. 60.

30 Cambolas (J. de), Le modele de la vie chrestienne où il est traité de la conformité que la vie du chrestien doibt avoir avec la Foy, et en particulier avec la personne, nom, doctrine et mysteres de Jésus-Christ Toulouse, A. Colomies, 1643, p. 170.

31 Bernières-Louvigny (J. de), Les œuvres spirituelles de Monsieur de Bernières-Louvigny..., Paris, Claude Cramoisy, 1670, première partie, p. 65.

32 Renty (G. de), Correspondance, op.cit., p. 186.

33 Vincent de Paul, Correspondance, entretiens, documents, édités par P. Coste, tome 9, Paris, 1923, pp. 56.

34 Laisné de la Marguerie (E.), Les véritables motifs, op.cit, p. 46.

35 Bernières-Louvigny (J. de), Les œuvres spirituelles, op.cit., première partie, p. 54.

36 Amelote (D.), La vie de sœur Marguerite du Saint-Sacrement, religieuse carmélite du monastere de Beaune..., Paris, Pierre le Petit, 1655, p. 384.

37 B.M. de Grenoble, ms. R5765, Actes de la Compagnie du Saint-Sacrement de Grenoble (1652-1666), $\mathrm{f}^{\circ} 17 \mathrm{v}^{\circ}$

38 Renty (G. de), Correspondance, op.cit., p. 461.

39 Noulleau (J.B.), L'Esprit du Christianisme dans le S. Sacrifice de la Messe, Paris, Charles du Mesnil, 1664, pp. 51.52.

40 Bernières-Louvigny (J. de), Le Chrestien interieur ou la conformité interieure que doivent avoir les chrestiens avec Jésus-Christ, par un solitaire, Paris Cramoisy, 1663, p. 263.

${ }^{41}$ Renty (G. de), Correspondance, op.cit, pp.699-70 r.

42 Bernières-Louvigny (J. de), Les œuvres spirituelles, op.cit, première partie, p. 39. 